\title{
Quantitative analysis of venation patterns of Arabidopsis leaves by supervised image analysis
}

\author{
Stijn Dhondt ${ }^{1,2}$, Dirk Van Haerenborgh ${ }^{1,2,3}$, Caroline Van Cauwenbergh ${ }^{1,2}$, Roeland M. H. Merks ${ }^{1,2,4,5}$, Wilfried Philips ${ }^{3}$, \\ Gerrit T. S. Beemster ${ }^{1,2,6, \dagger}$ and Dirk Inzé ${ }^{1,2, t, *}$ \\ ${ }^{1}$ Department of Plant Systems Biology, VIB, 9052 Gent, Belgium, \\ ${ }^{2}$ Department of Plant Biotechnology and Bioinformatics, Ghent University, 9052 Gent, Belgium, \\ ${ }^{3}$ Department of Telecommunications and Information Processing/Image Processing and Interpretation, \\ Interdisciplinary Institute for Broadband Technology, Ghent University, 9000 Gent, Belgium, \\ ${ }^{4}$ Centrum Wiskunde \& Informatica, 1098 XG Amsterdam, The Netherlands, \\ ${ }^{5}$ Netherlands Consortium for Systems Biology/Netherlands Institute for Systems Biology, 1098 XG Amsterdam, \\ The Netherlands, and \\ ${ }^{6}$ Department of Biology, University of Antwerp, 2020 Antwerpen, Belgium
}

Received 29 June 2011; revised 9 September 2011; accepted 27 September 2011.

*For correspondence (fax +32 9 3313809; e-mail dirk.inze@psb.vib-ugent.be).

${ }^{\dagger}$ These authors contributed equally to this work.

\begin{abstract}
SUMMARY
The study of transgenic Arabidopsis lines with altered vascular patterns has revealed key players in the venation process, but details of the vascularization process are still unclear, partly because most lines have only been assessed qualitatively. Therefore, quantitative analyses are required to identify subtle perturbations in the pattern and to test dynamic modeling hypotheses using biological measurements. We developed an online framework, designated Leaf Image Analysis Interface (LIMANI), in which venation patterns are automatically segmented and measured on dark-field images. Image segmentation may be manually corrected through use of an interactive interface, allowing supervision and rectification steps in the automated image analysis pipeline and ensuring high-fidelity analysis. This online approach is advantageous for the user in terms of installation, software updates, computer load and data storage. The framework was used to study vascular differentiation during leaf development and to analyze the venation pattern in transgenic lines with contrasting cellular and leaf size traits. The results show the evolution of vascular traits during leaf development, suggest a self-organizing mechanism for leaf venation patterning, and reveal a tight balance between the number of end-points and branching points within the leaf vascular network that does not depend on the leaf developmental stage and cellular content, but on the leaf position on the rosette. These findings indicate that development of LIMANI improves understanding of the interaction between vascular patterning and leaf growth.
\end{abstract}

Keywords: venation pattern, imaging software, vascular network, leaf development, cellular content, Arabidopsis.

\section{INTRODUCTION}

The vascular system of plants consists of a continuous network of vascular bundles (Esau, 1965). This network plays a crucial role in the mechanical support and transport of fluids within, from and towards leaves. The xylem is the main conduit for minerals and water, whereas the phloem ensures the transfer of dissolved photoassimilates. Both tissues comprise a number of specialized cell types, but all vascular cells differentiate from a single meristematic tissue, the procambium. Procambial cells are the first visible indication of specification during the vascular patterning process (Busse and Evert, 1999), and the phytohormone auxin has been identified as the initial signal triggering their 


\section{Stijn Dhondt et al.}

formation. Gradually restricted auxin transport routes, visualized by the expression domains of the PIN-FORMED1 (PIN1) auxin efflux carrier, define sites of procambium formation (Scarpella et al., 2006; Wenzel et al., 2007). During leaf development in Arabidopsis, this process produces a reticulate pattern that becomes more complex as the leaf develops (Candela et al., 1999; Scarpella et al., 2006; Wenzel et al., 2007). Secondary veins are joined in a series of prominent arches, leading to classification of Arabidopsis venation as brochidodromous (Hickey, 1988). Although many mutants with altered leaf venation patterns have been identified (Scarpella and Meijer, 2004; Sieburth and Deyholos, 2006), most have only been assessed qualitatively. The fact that vascular phenotypes have been identified visually suggests that perturbation of the vascular network in these mutants is relatively strong. Previous studies have demonstrated the usefulness of quantification of vascular traits, such as vein thickness, vein density, distributions of branching angles and vein lengths, and the number of branching points, free-ending veins and loops (Hill, 1980; Candela et al., 1999; Bohn et al., 2002; Steynen and Schultz, 2003; Kang and Dengler, 2004; Alonso-Peral et al., 2006; Brodribb et al., 2007; Boyce et al., 2009; Rolland-Lagan et al., 2009; Brodribb and Feild, 2010; Price et al., 2011). As manual analysis of these traits is very laborious and time-consuming, most analyses focused on part of the leaf. Recently, two enhanced methods have been described that allow quantification of the linear dimensions of vein patterns in entire leaves: a tool that allows manual vein pattern tracing (Rolland-Lagan et al., 2009) and a framework for manual vein extraction with built-in image analysis and drawing functions (Price et al., 2011). Both methods rely completely on user input, do not allow analysis of a batch of pictures, and are relatively time-consuming. Here, we introduce an online framework, Leaf Image Analysis Interface (LIMANI), for analysis of vascular networks in leaves, which automatically segments and measures venation patterns in a batch of cleared leaf images. The framework also includes a userfriendly interactive interface, enabling manual segmentation corrections for high-precision analysis. Using this framework, we investigated vascular differentiation throughout leaf development, and examined the role of cellular traits and leaf growth traits on vascular patterning in transgenic Arabidopsis lines.

\section{RESULTS AND DISCUSSION}

\section{Online analysis of vascular patterns using the interactive interface LIMANI}

Here we describe LIMANI, an online image analysis framework which includes the possibility of manual image segmentation corrections. The online approach allows complete freedom for the developer and has a number of advantages for the user: the tool does not require installa- tion, avoiding problems of operating system compatibility and software dependency, as encountered with image analysis libraries, and the user always has access to the most recent software version and can run the analysis on a remote server, preventing local overload of the user's machine and avoiding data loss by a back-up system. Furthermore, the framework was built in a modular fashion, making it relatively straightforward to plug different image processing and analysis algorithms into the same interface. Imaging tools often consist of two major parts: segmentation of an image for extraction of the content of interest, followed by measurement and calculation of specific traits. Thus, numerous applications within and beyond plant research may be integrated into LIMANI.

The LIMANI framework incorporates an image analysis algorithm for quantification of vascular patterns in Arabidopsis leaves. In this application, vascular networks are extracted from dark-field images of whole-mount, cleared leaves, and the extracted networks are quantified. Furthermore, the image analysis algorithm is implemented into an interactive user interface (Figure 1). To start, the user adds a new task or group by uploading images to the server and setting a number of image analysis parameters. Next, the user is directed to the task overview page, showing the status and parameter settings of all the user tasks in the database. Here, the user can compare and summarize the results of selected tasks or groups. Grouping biological replicates enables a quick comparison between genotypes by calculating mean values and SE for each genotype. Every task is also linked to its task detail page, which visually represents the extracted venation pattern and the values of the vascular parameters calculated from the extracted pattern. Furthermore, the user can re-start the segmentation algorithm with different initial parameter settings or review the extraction by making manual adjustments to the segmentation in a user-friendly manner. This latter possibility functions as a supervision and rectification step in the automated image analysis pipeline, making the analysis tool less of a 'black box' and ensuring that the output quality is in line with the user's expectations.

The review page contains an interactive canvas that allows editing and drawing of lines in the web interface, linking user-defined changes to the segmentation of the vascular network. The implemented drawing tools provide the opportunity to zoom in or out, scroll, change colors, select, move or remove complete vascular segments or parts thereof, draw free-ending segments and connect existing segments using a freehand or segmented linedrawing tool, and save corrections. 'Undo' and 're-do' functionalities are also implemented. In the review page, the segmentation consists of multiple layers that can be hidden or visualized. Combined with the input image displayed in the background, the extracted and actual networks can be compared quickly. After approval of the 


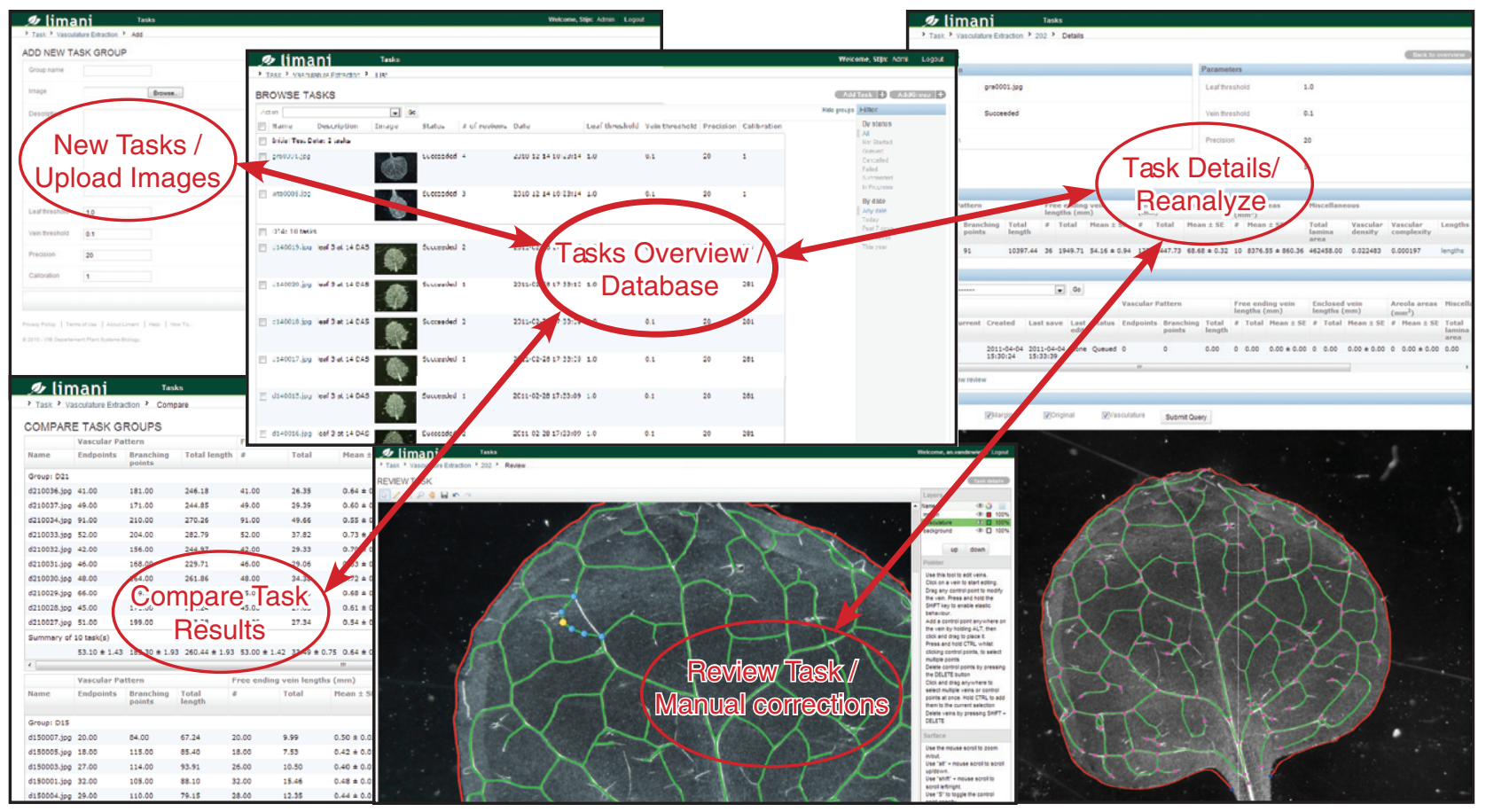

Figure 1. The LIMANI interface.

Screenshots represent the various web pages, which functions describe a schematic overview of the architecture of the framework.

adjusted segmentation, the vascular network is updated and sent to the server for quantification.

In essence, we have created an online interface to run image analysis algorithms for quantification of vascular networks in leaves, allowing us to control and rectify the automatically extracted patterns by incorporation of an interactive drawing application. This approach provides a fast, user-friendly and reliable method to analyze vascular networks in Arabidopsis leaves.

\section{Extraction of vascular networks}

The first and most important step is extraction of the vascular network. A gray-scale mathematical morphology image analysis algorithm (Dougherty and Lotufo, 2003) executes a sequence of functions on an input image (see below). The input image of choice is a dark-field picture of a single leaf cleared first in ethanol and then in lactic acid and mounted in lactic acid (Figure 2a). Dark-field microscopy creates a high contrast between the vascular strands and the background, primarily by light scattering on differentiated xylem vessels, and preferably an intermediate brightness within the leaf tissue. Starch accumulation in the leaf can complicate visualization of the vascular pattern, but clearing with chloral hydrate solution usually resolves this issue.

In the first image analysis step, the leaf is extracted from the background by recursive opening and closing of the input image using an increasing structuring element that results in an enhanced contrast between leaf and back- ground while preserving the global shape of the structure (Figure $2 b$ ). The leaf is then extracted by thresholding the image, in which the threshold is a user-defined fraction of the mean intensity of the entire image. When the segmentation yields more than one object, due to the presence of an air bubble for instance, the largest object is assumed to be the leaf. Possible holes in the resulting leaf mask are filled automatically. The intersection between this leaf mask and the original image results in removal of the background (Figure 2c). The leaf margin is obtained by subtraction of the leaf mask erosion from its dilation (Figure 2d).

The next step is extraction of the vascular pattern from the leaf. To remove the local background within the leaf, recursive opening and closing with an increasing structuring element is again applied (Figure 2e). In this case, the number of iterations is fewer than those for leaf segmentation to preserve local differences in background intensities. Subtraction of the resulting image from the leaf image results in a gray-scale extraction of the vascular pattern (Figure 2f). Gray-scale dilation, to highlight the venation, is followed by thresholding using an adjustable percentage of the maximum intensity of the image (Figures $2 g$ and S1a). Small structures disconnected from the vascular network are removed in a few steps, depending on their size and proximity to the network (Figures $2 \mathrm{~h}$ and $\mathrm{S} 1 \mathrm{~b}$ ) and the resulting binary image is thinned, after which small spurs are removed by end-point detection and recursive pruning of small segments (Figures $2 \mathrm{i}$ and $\mathrm{S} 1 \mathrm{c}$ ). At this point, the 

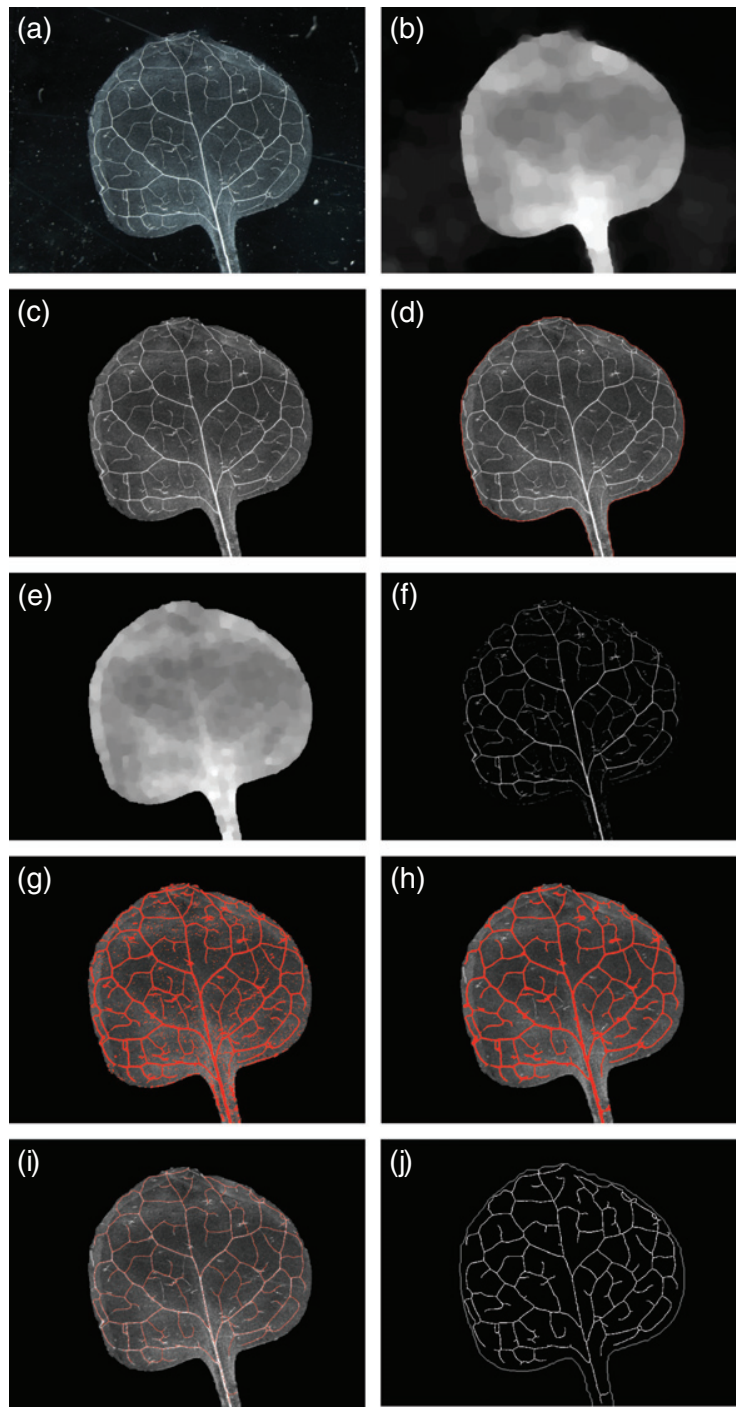

Figure 2. Automated extraction of the vascular network of an Arabidopsis leaf.

(a) Dark-field picture of a cleared leaf used as the input image for the analysis. (b) Contrast between the leaf and background enhanced by recursive opening and closing using an increasing structuring element.

(c) Extraction of the leaf from the background by thresholding the image in (b) using the mean intensity of the complete image.

(d) Detection of the leaf margin by subtraction of the eroded leaf mask from the dilated one.

(e) Application of recursive opening and closing using an increasing structuring element to remove the local background within the leaf.

(f) Subtraction of the image in (e) from the leaf image in (c) results in a grayscale extraction of the vascular pattern.

(g) Dilation of the pattern, followed by thresholding using an adjustable percentage of the maximum intensity of the image.

(h) Removal of small structures disconnected from the vascular network.

(i) Thinning of the resulting image, after which small spurs are removed.

(j) Segmentation completion by combination of the extracted leaf margin and the vascular pattern.

number of disconnected components is determined, and the vascular network is assumed to be the largest component. This step removes disconnected structures from the net- work, such as trichomes, that are often also illuminated by dark-field imaging. As a consequence, the algorithm is only suited for extraction of interconnected vascular networks. Hence, mutants such as $f k d 2 / s f c / v a n 3, \operatorname{trn} 1, \operatorname{trn} 2, \operatorname{cvp} 1$ and cvp2, which generate a vascular pattern consisting of vascular islands (Carland et al., 1999; Deyholos et al., 2000; Steynen and Schultz, 2003; Cnops et al., 2006), cannot be analyzed by the algorithm. The picture segmentation is completed by combining the extracted leaf margin and venation pattern by addition of the two images (Figure $2 \mathrm{j}$ ).

These steps represent the workflow by which the leaf margin and vascular network are segmented from a darkfield leaf image. Subsequently, the resulting pattern is quantified (see below), and, as mentioned before, is made available in the interactive drawing application for visual control, rectification and approval of the network, ensuring segmentation of the vascular pattern with the desired quality.

\section{Quantification of vascular traits}

Once the vascular network and leaf margins have been extracted, the next step is to quantify the vascular parameters. The actual measurements are implemented in a second algorithm for which the input is the vascular pattern and leaf margin obtained using the first algorithm (Figure 3a). The algorithm is partly derived from an approach developed previously for analysis of in vitro vascular networks in human endothelial cell cultures (Mezentzev et al., 2005; Merks et al., 2006). First, the leaf lamina is defined and separated from the petiole in a standardized manner to avoid the possibility that petiole length affects leaf size and vascular measurements. The leaf lamina is separated by recursive dilation of the binary leaf margin image, closing the petiole (Figure $3 b$ ). Recursive thinning of this object results in a closed object, excluding most of the petiole (Figure 3c). The area enclosed by both this object and the leaf margin defines the leaf lamina. The vascular network to be analyzed is reduced to the part inside the leaf lamina by taking the intersection of both (Figure 3d). Because the segmentation algorithm extracts the vascular pattern as a four-connected skeletonized line, branching points and endpoints in the network can be identified as pixels with three or four neighbors and those with only one neighbor, respectively (Figure 3e). Points positioned on vascular strands have two neighbors. Branching points that are in close proximity to each other (fewer than seven pixels) are considered as a single branching point because this is how it would be defined by visual recording. Subtraction of branching points from the vascular pattern divides the network into disconnected lines, hereafter called vascular elements. The network is then represented as a graph structure, defining which branching points and end-points are connected to which vascular elements, and the topology of each vascular element is defined by its coordinates. The above 
Figure 3. Quantification of the vascular network (a) Binary image comprising the leaf margin and the vascular network extracted by the first algorithm.

(b) Dilation of the leaf margin, closing the petiole. For visualization, the original input image is used as the background image.

(c) Determination of the leaf lamina.

(d) Indication of the leaf area (red) and the vascular network covering the leaf lamina.

(e) Visualization of the branching points (green), end-points (blue) and vascular segments (red).

(f) Visualization of the areolas, which are randomly color-coded.
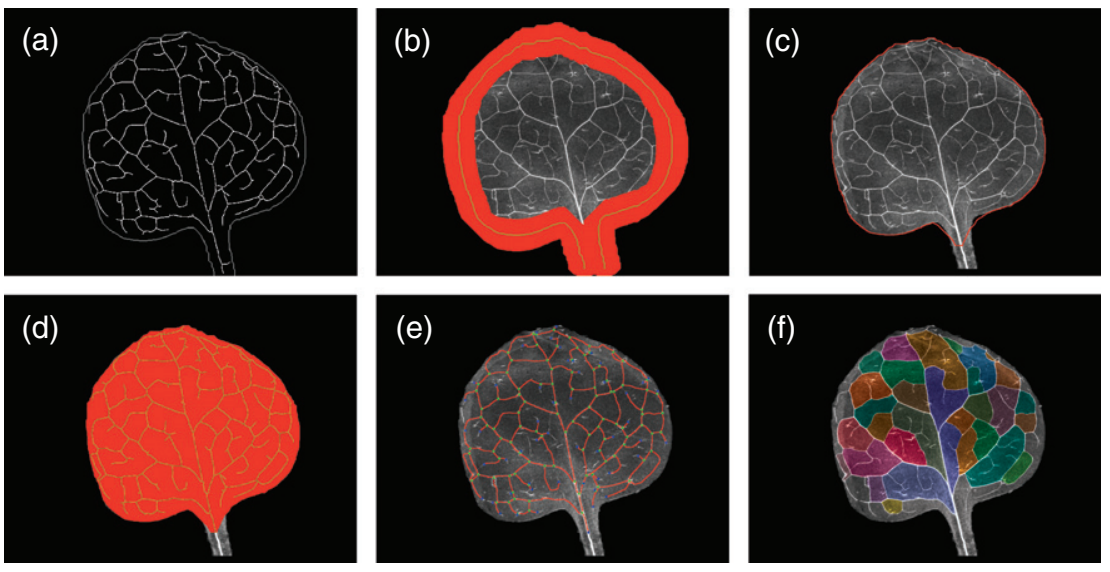

transformations allow a more mathematical and faster processing of the data. To measure vein lengths, the detected vascular elements are sub-divided into line sections of user-defined length. The length of a vein is calculated as the sum of the Euclidean distances between the end-points of the line sections. The total length of the vascular system in the leaf is the sum of the lengths of each vascular element. The graph structure allows determination of whether a vascular element is connected to an end-point or links two branching points, enabling calculation of the mean length of the free-ending and enclosed veins. Furthermore, LIMANI provides an output list that includes the lengths of all individual vascular elements. The areoles (areas in the lamina surrounded by vascular strands) are also extracted from the graph structure, and their number and mean areas are calculated (Figure 3f). All length and area measurements are calibrated from pixels to standard units based on a userdefined calibration value. Finally, vascular density (vein length per unit area) and complexity (sum of the number of end-points, branching points and vascular elements) are calculated. The number of branching points has been used previously to represent vascular complexity (Hamada et al., 2000; Jun et al., 2002). Kang et al. (2007) remarked correctly that the number of end-points provides additional information on the complexity of a venation network. Adding the term 'number of vascular elements' to the equation allows discrimination between networks that show an altered ratio between three-way and four-way junctions at vascular branching points, giving a complete representation of the factors affecting vascular complexity. Hence, LIMANI determines ten measures of the vascular network starting from a dark-field cleared leaf picture, providing quantitative values for the biologically most relevant vascular traits (Table S1).

\section{Vascular differentiation throughout leaf development}

Using LIMANI, we analyzed development of the venation pattern in the third leaf of Arabidopsis (Col-0) to examine evolution of the vascular features during leaf growth. Leaves were analyzed at $11,12,13,14,15,17,19$, and 21 days after stratification (DAS) ( $n \geq 10$ ). The analysis started after differentiation of the midvein, which was only apparent in $20 \%$ of the samples at 10 DAS. This timing implies that the vascular differentiation process starts at the transition from primary to secondary leaf morphogenesis, which also occurs at approximately day 10 (Andriankaja M., Dhondt S., De Bodt S., Inzé D., unpublished data). Early during this period, the leaf lamina grows exponentially, with a progressive decrease in the relative expansion rate after day 14 (Figure 4a), while the vascular pattern length increases at a constant (exponential) rate from day 11 to 14 , after which the rate of increase gradually declines (Figure 4b). Furthermore, the vascular density increased to $3.2 \mathrm{~mm} \mathrm{~mm}^{-2}$ at 14 DAS, and then rapidly decreased to $1.7 \mathrm{~mm} \mathrm{~mm}^{-2}$ (Figure 4c), indicating that the vascular pattern extends faster than growth of the leaf blade during the exponential growth phase, but undergoes a slower increase later during development. This developmental trend in vascular density is comparable to that previously identified in juvenile and more adult rosette leaves of Arabidopsis (Candela et al., 1999; Kang and Dengler, 2004; Rolland-Lagan et al., 2009). Interestingly, the vein density decreases and increases in leaves formed later during rosette development in Columbia (Col) and Landsberg erecta (Ler) backgrounds, respectively. Moreover, the corresponding density values in Col background are generally lower than those in Ler. Measurements of leaf 3 show that densities of branching points and endpoints also peak at day 14, i.e. simultaneously with the vascular density (Figure S2), and this is also the time at which the mean length of the free-ending veins is minimal (Figure 4d). Three days later, at 17 DAS, the topology of the vascular pattern is complete, as reflected by the the vascular complexity (Figure $4 \mathrm{~h}$ ) and the plateau reached for the number of end-points, branching points and areoles (Figure $4 \mathrm{e}-\mathrm{g}$ ). In other words, the increase in total vein length at 17 DAS is primarily due to extension of the existing connections in the pattern. Interestingly, the numbers of end-points and branching points are strongly correlated (Figure 4i). A linear regression analysis with the intercept at 


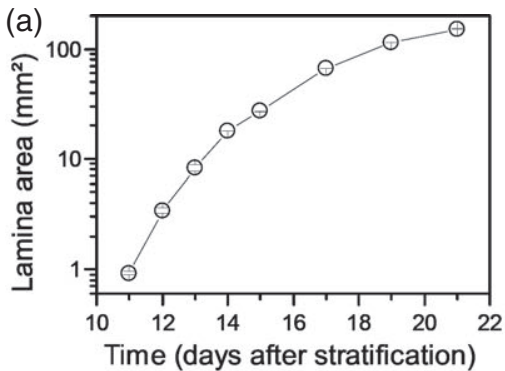

(c)

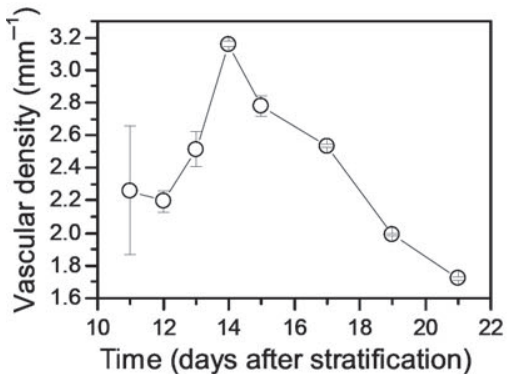

(e)

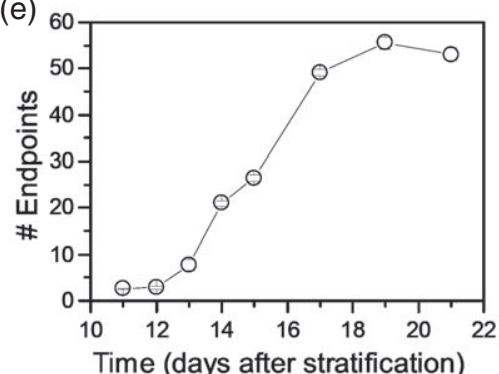

(g)
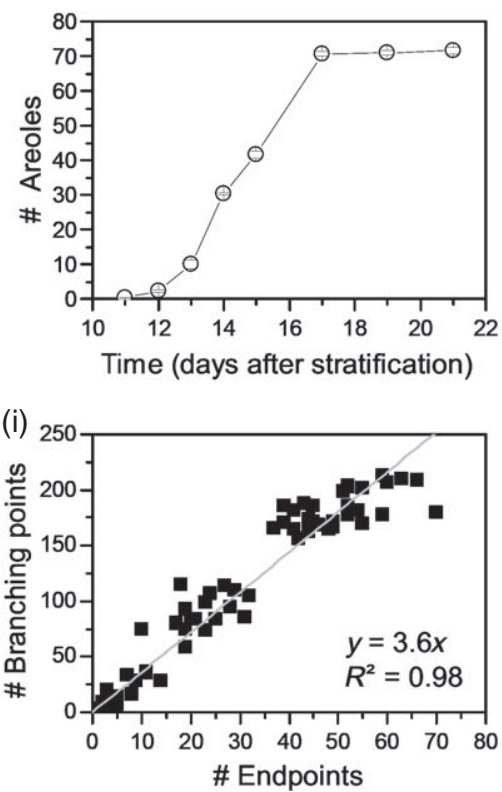

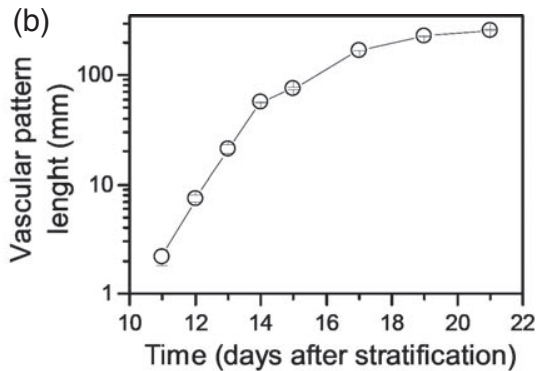

(d)

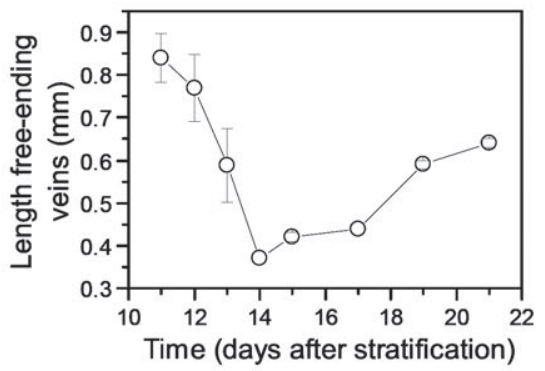

(f)

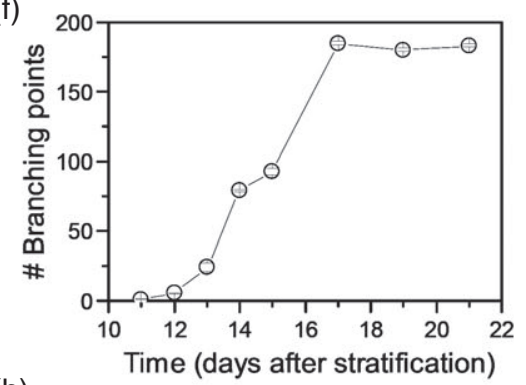

(h)
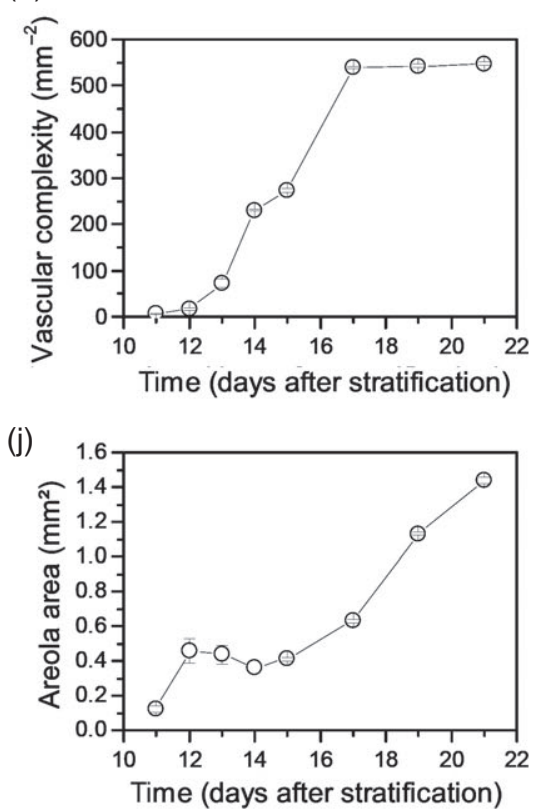

Figure 4. Time-lapse analysis of vascular differentiation during leaf development.

Venation patterns of third-node Arabidopsis $\mathrm{Col}-$ 0 leaves were analyzed at $11,12,13,14,15,17,19$ and 21 days after stratification (DAS).

(a) Lamina area

(b) Vascular pattern length.

(c) Vascular density.

(d) Length of free-ending veins

(e) Number of end-points.

(f) Number of branching points.

(g) Number of areoles.

(h) Vascular complexity.

(i) Correlation between the numbers of endpoints and branching points by linear regression with the intercept at the origin.

(j) Areola area.

Error bars represent SE $(n \geq 10)$. the origin results in an $R^{2}$ value of 0.98 for $y=3.6 x$, indicating that, for every vascular end-point, a mean of 3.6 branching points are present in the leaf, independently of the developmental stage. This observation suggests a bal- ance between formation of free-ending veins and connection of veins to the existing pattern. Furthermore, during the period of rapid pattern extension, from days 12-15, the mean areola area is relatively constant (Figure $4 \mathrm{j}$ ) but the 
number of end-points, branching points and areoles expands rapidly (Figure $4 \mathrm{e}-\mathrm{g}$ ). Thus, the mean number of pattern elements per unit area, i.e. the scaling of the pattern, remains approximately constant during rapid tissue expansion. Such a typical pattern scale is consistent with selforganization hypotheses for leaf venation patterning, in which motile chemical signals (Meinhardt, 1976), including auxin (Dimitrov and Zucker, 2006; Merks et al., 2007), or mechanical strain (Couder et al., 2002; Laguna et al., 2008) maintain a constant distance between newly formed pattern elements. Leaf growth creates more space between the permanently differentiated veins, driving further expansion of the network, but not necessarily meaning that areole areas are uniform across the leaf. A gradient in areole sizes from the centre of the leaf to the leaf margin has been reported (Rolland-Lagan et al., 2009). Larger areole areas near the middle of the leaf may be indicative of a stronger sink strength of the midvein compared to the higher order veins or may represent local growth differences.

Taken together, analysis of vascular differentiation during leaf development using the LIMANI framework provides a better insight into the patterning process. It showed that active expansion of the differentiated venation starts at the transition from primary to secondary leaf morphogenesis and ends before the shutdown of cell expansion. Furthermore, our analysis revealed a balance between the formation of new veins and the connection of veins to the existing pattern, and suggests a self-organizing mechanism for leaf venation patterning, followed by uniform expansion of a terminally differentiated pattern.

\section{Cellular traits affect vascular patterning}

In addition to analysis of vascular development in wild-type plants, we assessed whether cell density and leaf size affect vascularization. To this end, we studied the vascular topology in six transgenic lines with contrasting cellular and leaf growth traits that have been described previously. These lines included the grandifolia mutants gra2-D and gra3-D, which contain a large segmental duplication in the lower part of chromosome 4 (Horiguchi et al., 2009), an overexpression line for the CYCLIN D2; 1 gene (35S::CYCD2;1) ( $\mathrm{Qi}$ and John, 2007), the SHORT-ROOT and SCARECROW mutants shr-6 and scr-3 (Fukaki et al., 1996; Gallagher et al., 2004), and an over-expression line for the cell-cycle inhibitor KIP-RELATED PROTEIN 2 (35S::KRP2) (De Veylder et al., 2001). Analysis of the vascular pattern of these lines was used for a correlation study between vascular parameters and leaf and cell growth traits. To obtain a better understanding of the effect of foliar cellular content on the vein patterning process, we determined the final leaf size, mesophyll cell area and mesophyll cell number per leaf in first-node leaves of the six transgenic lines, and compared them to the wild-type measurements at 24 DAS (Table 1). These cellular measurements were repeated on additional leaves at 8 DAS, early during vascular patterning of the firstnode leaf pair (data not shown), allowing calculation of the number of cells produced per cell between 8 and 24 DAS (Table 1). The latter measurement was used as a proxy for the length of the cell proliferation phase in the leaf. The rationale behind the use of this numerical proxy is that 8 DAS is the time point at which the cell-cycle arrest front is usually established in leaves 1 and 2 of Arabidopsis, and that the leaves stop growing and reach a mature developmental stage at 24 DAS. The number of cells produced per cell between 8 and 24 DAS relates to the number of cell divisions between establishment of the cell-cycle arrest front and maturity on an individual cell basis, and thus serves as a reliable proxy for the length of the proliferation phase.

gra2-D and gra3-D produced large leaves primarily due to an increase in cell number as a consequence of an extended cell proliferation phase. This phenotype is more pronounced in gra3- $D$ than in gra2- $D$, as reported previously (Horiguchi et al., 2009). In the CYCD2;1 over-expression line, the cell number increased twofold, accompanied by a similar decrease in cell size, without a drastic effect on the final leaf size (Qi and John, 2007). The leaves of scr, shr and 35S::KRP2

Table 1 Vascular analysis of wild-type plants and six transgenic lines with contrasting cellular and leaf size phenotypes at 24 DAS

\begin{tabular}{|c|c|c|c|c|}
\hline Genotype & Leaf area $\left(\mathrm{mm}^{2}\right)$ & Cell area $\left(\mu \mathrm{m}^{2}\right)^{\mathrm{a}}$ & Cell number ${ }^{b}$ & $\begin{array}{l}\text { Number of cell } \\
\text { divisions per cell } \\
\text { between days } 8 \text { and } 24^{c}\end{array}$ \\
\hline gra3-D & $44.66 \pm 2.92$ & $1215 \pm 41$ & $36741 \pm 4117$ & $15.27 \pm 2.87$ \\
\hline gra2-D & $33.06 \pm 2.40$ & $1488 \pm 97$ & $22217 \pm 2349$ & $10.88 \pm 1.64$ \\
\hline Wild-type & $18.75 \pm 0.54$ & $1313 \pm 36$ & $14276 \pm 1365$ & $3.68 \pm 0.54$ \\
\hline 35S::CYCD2;1 & $15.76 \pm 0.66$ & $636 \pm 73$ & $24616 \pm 3031$ & $6.09 \pm 0.96$ \\
\hline$s c r-3$ & $8.21 \pm 0.45$ & $1128 \pm 84$ & $7278 \pm 582$ & $3.64 \pm 0.60$ \\
\hline shr-6 & $4.40 \pm 0.29$ & $1065 \pm 78$ & $4130 \pm 467$ & $1.20 \pm 0.19$ \\
\hline 35S::KRP2 & $2.78 \pm 0.20$ & $1845 \pm 167$ & $1506 \pm 274$ & $1.83 \pm 0.46$ \\
\hline
\end{tabular}

\footnotetext{
${ }^{a}$ Area of mesophyll cells in the sub-epidermal layer observed in a paradermal view.

${ }^{\mathrm{b}}$ Total number of mesophyll cells estimated by dividing leaf area by cell density.

${ }^{\mathrm{c}}$ Number of cell divisions per cell between 8 and 24 DAS calculated by dividing cell number at day 24 by that at day 8 .

The genotypes are ranked according to their final leaf size. Values are means $\pm \operatorname{SE}(n \geq 5)$.
} 


\section{Stijn Dhondt et al.}

lines were smaller than those of the wild-type. In the scr and shr mutants, the reduction was mainly due to a decrease in cell number, caused by a longer cell-cycle duration, together with an earlier exit from the cell proliferation phase in the case of $s h r$, explaining its more drastic phenotype (Dhondt et al., 2010). In the 35S::KRP2 line, the extreme reduction in cell number was partially compensated for by a strongly increased cell size (De Veylder et al., 2001). Leaves at 24 DAS were also utilized to analyze the vascular network by means of LIMANI. Vascular measures were obtained for all lines (Table S1), and correlations with leaf area, cell area, cell number and cell production per cell between days 8 and 24 corresponding to the duration of the cell-proliferation phase, were calculated for each of the vascular parameters (Figure 5a). The strongest correlation for most vascular parameters was found with the leaf area. Among these, the highest correlation was between leaf area and the total length of the vascular pattern $\left(R^{2}=0.98\right)$ (Figure $\left.5 \mathrm{~b}\right)$. This

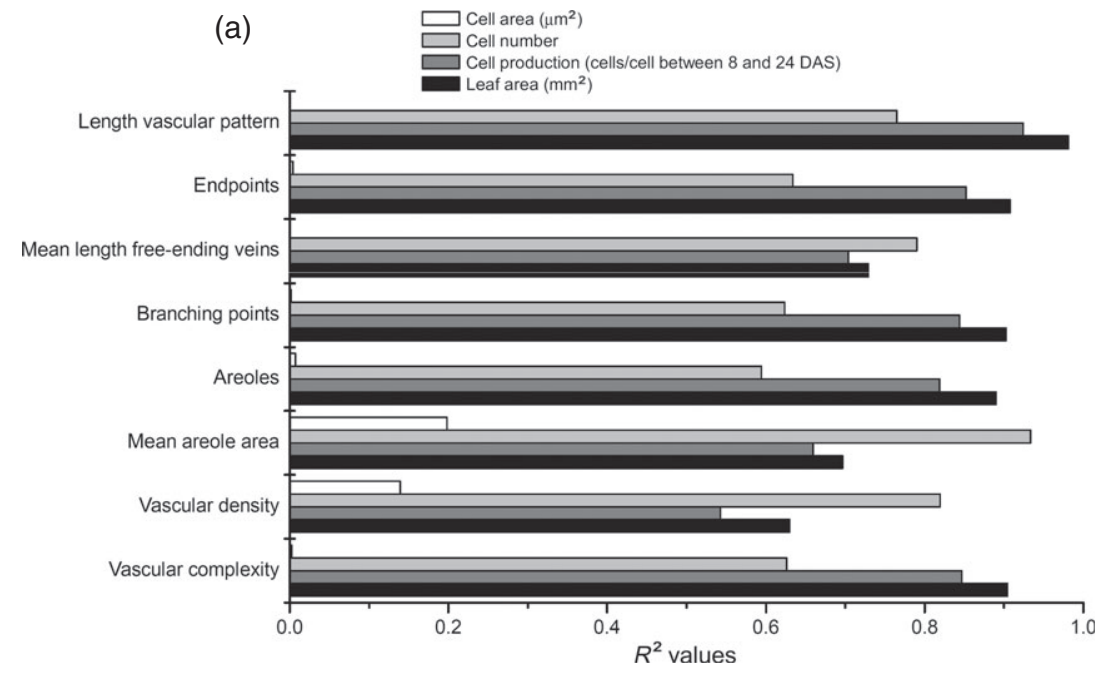

(b)

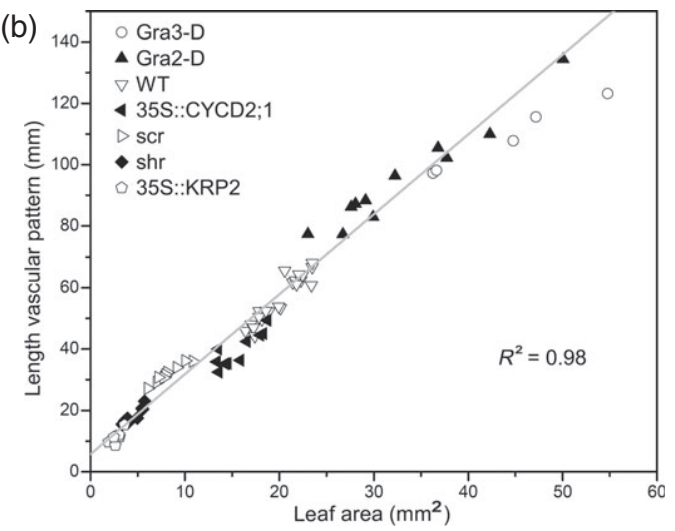

(c)

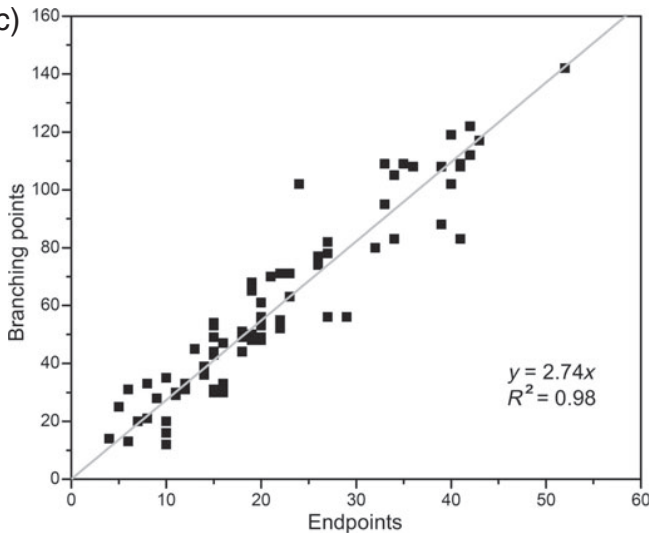

Figure 5. Correlation study between cellular traits and vascular parameters.

Correlations were determined by linear regression analysis using measurements from wildtype plants and six transgenic lines with contrasting cellular and leaf size traits

(a) Correlations between cell area, cell number, cell production and leaf area, and differentiated vascular pattern traits in mature leaves. Cell production, expressed as the number of cells produced per cell between 8 and 24 DAS, is used as a proxy for the length of the cell proliferation phase.

(b) Correlation between leaf area and total length of the vascular pattern.

(c) Correlation between the numbers of endpoints and branching points by linear regression with the intercept at the origin. 
suggests that formation of the vascular network and the space available within the leaf are tightly linked, in accordance with a self-organizing mechanism for pattern formation. The selected transgenic lines, together with the wild-type, covered a large range of leaf sizes, from 2 to $55 \mathrm{~mm}^{2}$ (Figure $5 \mathrm{~b}$ ). The length of the proliferation phase was also strongly correlated with most vascular parameters. The fact that cell production is the only indirect measurement, derived from the difference between samples at 8 and 24 DAS, in this correlation analysis underlines the strength of this relationship even more. This finding confirms that modifications of cell proliferation patterns are correlated with changes in vein complexity (Kang et al., 2007), and is in line with the correlation found between termination of vein formation and mesophyll differentiation (Scarpella et al., 2004). However, in our data set, the duration of cell proliferation and final leaf size were also very well correlated $\left(R^{2}=0.95\right)$, making it difficult to separate the effect of the length of the cell proliferation phase and overall leaf growth on development of the vascular network. For the mean length of the free-ending veins, vascular density and the mean areole area, the highest correlation was found with total cell number in the leaf (Figure 5a). Furthermore, vascular density and mean areole area were the only parameters that significantly correlated with the mesophyll cell area (Figure 5a). Analysis of vascular patterning during leaf growth revealed that the number of areoles stabilized while the leaf was still growing (Figure $4 a, g$ ), suggesting that expansion of the cells enclosed by the areole drove the increase in areole area during the later stages of leaf development. The more cells enclosed by the areole at that stage, the larger the net effect of expansion of the individual cells, explaining the observed correlations with the areole area. The same reasoning can explain the negative correlation with vascular density: the more cells in the leaf, the higher the net impact of expansion of the individual cells on enlargement of the network and the less dense the network will be at maturity. Moreover, the time window during which this process occurs has already started at shutdown of the vascular patterning, which is earlier during leaf development than observation of a stabilized differentiated pattern (17 DAS). Another interesting observation is the strong correlation between the numbers of end-points and branching points (Figure $5 \mathrm{c}$ ). A linear regression analysis with the intercept at the origin resulted in an $R^{2}$ value of 0.98 for $y=2.74 x$, indicating that the balance does not depend on the foliar cellular content. In comparison with leaf 3 from the developmental study, leaf 2 contains fewer branching points per end-point. A significant deviation from this constant is found for transgenic lines that are affected in the connection of vascular strands to pre-existing vascular bundles, such as ron 1/fry1/sal1, fkd2/sfc/van3 and cvp2, which have an open vascular pattern as a consequence (Steynen and Schultz, 2003; Carland and Nelson, 2004; Robles et al., 2010).
These correlation studies reveal that many vascular phenotypes are linked to developmental changes. As vein patterning and growth occur concurrently, these processes are closely related. A shift in the developmental timing of cell proliferation or cell expansion, or a change in the cell division or cell expansion rate, will have a significant impact on a number of vascular parameters in the leaf. Hence, the observed vascular phenotype may be a primary as well as a secondary effect of the perturbation. In addition, a strong link between vascular patterning and leaf shape has been observed. Nevertheless, the question remains whether a change in leaf shape is the cause or consequence (or both) of the change in vascular patterning (Dengler and Kang, 2001; Runions et al., 2005; Fujita and Mochizuki, 2006). As vascular differentiation occurs relatively late in development, we assume that the influence of a fully functional and differentiated venation pattern on leaf shape and growth is rather limited, especially because differentiation of the midvein takes places near the end of the cell proliferation phase, the timing of which is strongly correlated with the final leaf size. This indicates that growth factors delivered locally by the differentiated vascular bundles probably do not contribute much to the final leaf size. However, at the time of vascular patterning, while the leaf is still proliferating, the developing vasculature also transports growth factors within the leaf, including auxin. The transport of these factors can affect local cell division and expansion, suggesting that removal of growth factors by the developing venation pattern may have more impact on leaf growth than their supply by the differentiated network. Nevertheless, the positive correlation of leaf vein density with the photosynthetic rate supports the hypothesis that vein positioning limits photosynthesis via its influence on foliar hydraulic efficiency (Brodribb et al., 2007). Thus, a high vascular density in old leaves, acting as a source tissue, may increase the production of photoassimilates, which, after transportation, may drive growth in young leaves, acting as a sink tissue. A careful analysis of leaf growth in various mutants or ecotypes with an altered vascular density may resolve this question.

In conclusion, the online framework LIMANI was developed for analysis of vascular networks in leaves, allowing automatic segmentation and measurement of venation patterns and including an interactive interface for manual segmentation corrections. This tool enables more quantitative analysis of vascular mutants and is able to generate data that are necessary to test various theoretical and mathematical models describing the underlying mechanisms of vascular patterning (Couder et al., 2002; Rolland-Lagan and Prusinkiewicz, 2005; Runions et al., 2005; Dimitrov and Zucker, 2006; Feugier and Iwasa, 2006; Merks et al., 2007; Laguna et al., 2008). We used the LIMANI framework to study vascular differentiation during leaf development and to analyze the venation pattern in transgenic lines with 
contrasting cellular and leaf growth traits. The results show the evolution of vascular traits during leaf development, and suggest a self-organizing mechanism for leaf venation patterning. We examined the effect of cellular content and leaf growth on vein patterning, and revealed a tight balance between the number of end-points and branching points within the leaf vascular network, independent of the leaf developmental stage and cellular content, but dependent on the position of the leaf on the rosette. These findings indicate that use of LIMANI can lead to a better understanding of the interaction between two processes occurring concurrently in leaf development, i.e. vascular patterning and leaf growth.

\section{EXPERIMENTAL PROCEDURES}

\section{Plant materials and growth conditions}

Arabidopsis thaliana (L.) Heyhn. was used as the wild-type, and transgenic gra2-D, gra3-D (Horiguchi et al., 2009), 35S::CYCD2;1 (Qi and John, 2007), shr-6 (Gallagher et al., 2004), scr-3 (Fukaki et al., 1996), and 35S::KRP2 (De Veylder et al., 2001) plants were included in the analysis of how cellular traits affect vascular patterning. These lines were all in the Columbia (Col) background. Seeds were sterilized in a $3 \% \mathrm{NaClO}$ solution (Carl Roth, http://www.carlroth.com/) for $15 \mathrm{~min}$, and sown on medium containing half-strength Murashige and Skoog medium (Duchefa, http://www.duchefa.com/) solidified with $0.9 \mathrm{~g} \mathrm{I}^{-1}$ plant tissue culture agar (Lab M, http:// www.labm.com/) on round plates (Becton-Dickinson, http://www .bd.com/). After a stratification period of 2 days, the plates were placed in a growth chamber under long-day conditions ( $16 \mathrm{~h}$ light/ $8 \mathrm{~h}$ darkness) at $22^{\circ} \mathrm{C}$ with a light intensity of $80-100 \mathrm{mE} \mathrm{m}{ }^{-2} \mathrm{sec}^{-1}$ supplied by cool-white fluorescent tubes (Spectralux Plus 36W/840; Radium, http://www.radium.de/en/).

\section{Sample preparation and imaging}

Leaves were harvested, cleared in $100 \%$ ethanol, and subsequently cleared and stored in lactic acid for microscopy. In cases of strong starch accumulation, the leaves were briefly cleared in a solution of $80 \mathrm{~g}$ chloral hydrate, $20 \mathrm{ml}$ glycerol and $10 \mathrm{ml}$ water. The cleared leaves were whole-mounted on slides in lactic acid and studied using a dark-field MZ16 stereomicroscope (Leica, http://www.leicamicrosystems.com/). To overcome highlighting of scratches on the images, the glass object surface was replaced with the plastic lid of a square $9 \times 9 \mathrm{~cm}$ Petri dish (Greiner Bio-One, http://www.greinerbioone.com/). Images were captured using a DS-5Mc camera (Nikon, http://www.nikon.com/) mounted on the stereomicroscope.

\section{Image analysis and framework set-up}

The Leaf Image Analysis Interface (LIMANI) is available online at http://limani.psb.ugent.be/, and is supported by the current versions of the Firefox, Google Chrome, and Safari web browsers. Demonstration videos that illustrate the functionality of the framework, FAOs, and user support are available online. The image analysis algorithms were written in Python, using the SDC Morphology Toolbox for Python (http://www.mmorph.com/pymorphpro/). The web interface was built using standards-compliant HTML5, with Java script to enable greater interaction with the user and making use of the Google Closure Library (http://code.google.com/closure/ library/). All web pages are served by the Python Django web framework (http://www.djangoproject.com/) on top of Apache (http://www.apache.org/). Static data (e.g. images or scripts) are served by Apache as is. Celery was chosen as the task queue server (http://celeryproject.org/). AMOP messaging was utilized to distribute tasks among multiple computers and multiple processes. The web server is incorporated into the data protection protocol of the Department of Plant Systems Biology, where the server is located.

\section{ACKNOWLEDGEMENTS}

We thank the members of the 'Systems Biology of Yield' research group at the department of Plant Systems Biology (VIB) and the members of the AGRON-OMICS consortium for testing and providing feedback on the LIMANI framework. This work was supported by grants from the Interuniversity Attraction Poles Program (Belgian Network BARN: 'Growth and Development of Higher Plants'; IUAP $\mathrm{VI} / 33$ ), initiated by the Science Policy Office of the Belgian State, the 'Bijzonder Onderzoeksfonds Methusalem project' (number BOF08/ 01M00408) of Ghent University, the European Community 6th Framework Programme ('AGRON-OMICS', LSHG-CT-2006-037704), and Marie Curie Intra-European Fellowship MEIF-CT-2005-025084 and Marie Curie European Reintegration Grant PERG03-GA-2008230974 (to R.M.), and was co-financed by the Netherlands Consortium for Systems Biology, which is part of the Netherlands Genomics Initiative/Netherlands Organization for Scientific Research. S.D. is indebted to the Agency for Innovation by Science and Technology for a pre-doctoral fellowship.

\section{SUPPORTING INFORMATION}

Additional Supporting Information may be found in the online version of this article:

Figure S1. Detailed representation of venation segmentation steps. Figure S2. Densities of branching points and end-points during leaf development.

Table S1. Vascular analysis of wild-type plants and six transgenic lines, with contrasting cellular and leaf size phenotypes at 24 DAS. Please note: As a service to our authors and readers, this journal provides supporting information supplied by the authors. Such materials are peer-reviewed and may be re-organized for online delivery, but are not copy-edited or typeset. Technical support issues arising from supporting information (other than missing files) should be addressed to the authors.

\section{REFERENCES}

Alonso-Peral, M.M., Candela, H., del Pozo, J.C., Martínez-Laborda, A. Ponce, M.R. and Micol, J.L. (2006) The HVE/CAND1 gene is required for the early patterning of leaf venation in Arabidopsis. Development, 133, 3755-3766.

Bohn, S., Andreotti, B., Douady, S., Munzinger, J. and Couder, Y. (2002) Constitutive property of the local organization of leaf venation networks. Phys. Rev. E, 65, 061914.

Boyce, C.K., Brodribb, T.J., Feild, T.S. and Zwieniecki, M.A. (2009) Angio sperm leaf vein evolution was physiologically and environmentally transformative. Proc. R. Soc. Lond. B Biol. Sci. 276, 1771-1776.

Brodribb, T.J. and Feild, T.S. (2010) Leaf hydraulic evolution led a surge in leaf photosynthetic capacity during early angiosperm diversification. Ecol. Lett. 13, 175-183.

Brodribb, T.J., Feild, T.S. and Jordan, G.J. (2007) Leafmaximum photosynthetic rate and venation are linked by hydraulics. Plant Physiol. 144, 1890-1898.

Busse, J.S. and Evert, R.F. (1999) Pattern of differentiation of the first vascular elements in the embryo and seedling of Arabidopsis thaliana. Int. J. Plant Sci. 160, 1-13.

Candela, H., Martínez-Laborda, A. and Micol, J.L. (1999) Venation pattern formation in Arabidopsis thaliana vegetative leaves. Dev. Biol. 205, 205-216.

Carland, F.M. and Nelson, T. (2004) COTYLEDON VASCULAR PATTERN2 mediated inositol $(1,4,5)$-triphosphate signal transduction is essential for closed venation patterns of Arabidopsis foliar organs. Plant Cell, 16, 12631275. 
Carland, F.M., Berg, B.L., FitzGerald, J.N., Jinamornphongs, S., Nelson, T. and Keith, B. (1999) Genetic regulation of vascular tissue patterning in Arabidopsis. Plant Cell, 11, 2123-2137.

Cnops, G., Neyt, P., Raes, J. et al. (2006) The TORNADO1 and TORNADO2 genes function in several patterning processes during early leaf development in Arabidopsis thaliana. Plant Cell, 18, 852-866.

Couder, Y., Pauchard, L., Allain, C., Adda-Bedia, M. and Douady, S. (2002) The leaf venation as formed in a tensorial field. Eur. Phys. J. B, 28, 135-138.

De Veylder, L., Beeckman, T., Beemster, G.T.S., Krols, L., Terras, F., Landrieu, I., Van Der Schueren, E., Maes, S., Naudts, M. and Inzé, D. (2001) Functiona analysis of cyclin-dependent kinase inhibitors of Arabidopsis. Plant Cell, 13, 1653-1667.

Dengler, N. and Kang, J. (2001) Vascular patterning and leaf shape. Curr. Opin. Plant Biol. 4, 50-56.

Deyholos, M.K., Cordner, G., Beebe, D. and Sieburth, L.E. (2000) The SCARFACE gene is required for cotyledon and leaf vein patterning. Development, 127, 3205-3213.

Dhondt, S., Coppens, F., De Winter, F., Swarup, K., Merks, R.M.H., Inzé, D., Bennett, M.J. and Beemster, G.T.S. (2010) SHORT-ROOT and SCARECROW regulate leaf growth in Arabidopsis by stimulating S-phase progression of the cell cycle. Plant Physiol. 154, 1183-1195.

Dimitrov, P. and Zucker, S.W. (2006) A constant production hypothesis guides leaf venation patterning. Proc. Natl Acad. Sci. USA, 103, 9363-9368.

Dougherty, E.R. and Lotufo, R.A. (2003) Hands-on Morphological Image Processing. Bellingham, WA: International Society for Optical Engineering

Esau, K. (1965) Plant Anatomy, 2nd edn. New York: John Wiley \& Sons.

Feugier, F.G. and Iwasa, Y. (2006) How canalization can make loops: a new model of reticulated leaf vascular pattern formation. J. Theor. Biol. 243, 235-244.

Fujita, H. and Mochizuki, A. (2006) The origin of the diversity of leaf venation pattern. Dev. Dyn. 235, 2710-2721.

Fukaki, H., Fujisawa, H. and Tasaka, M. (1996) SGR1, SGR2, and SGR3: nove genetic loci involved in shoot gravitropism in Arabidopsis thaliana. Plant Physiol. 110, 945-955.

Gallagher, K.L., Paquette, A.J., Nakajima, K. and Benfey, P.N. (2004) Mechanisms regulating SHORT-ROOT intercellular movement. Curr. Biol. 14, 1847-1851.

Hamada, S., Onouchi, H., Tanaka, H., Kudo, M., Liu, Y., Shibata, D., Machida, C. and Machida, Y. (2000) Mutations in the WUSCHEL gene of Arabidopsis thaliana result in the development of shoots without juvenile leaves. Plant J. 24, 91-101.

Hickey, L.J. (1988) A revised classification of the architecture of dicotyledonous leaves. In Anatomy of the Dicotyledons, Volume 1, 2nd edn (Metcalfe, C.R. and Chalk, L., eds). Oxford: Clarendon Press, pp. 25-39.

Hill, R.S. (1980) A numerical taxonomic approach to the study of angiosperm leaves. Bot. Gaz. 141, 213-229.

Horiguchi, G., Gonzalez, N., Beemster, G.T.S., Inzé, D. and Tsukaya, H. (2009) Impact of segmental chromosomal duplications on leaf size in the gran difolia-D mutants of Arabidopsis thaliana. Plant J. 60, 122-133.

Jun, J.H., Ha, C.M. and Nam, H.G. (2002) Involvement of the VEP1 gene in vascular strand development in Arabidopsis thaliana. Plant Cell Physiol. 43 323-330.
Kang, J. and Dengler, N. (2004) Vein pattern development in adult leaves of Arabidopsis thaliana. Int. J. Plant Sci. 165, 231-242.

Kang, J., Mizukami, Y., Wang, H., Fowke, L. and Dengler, N.G. (2007) Modification of cell proliferation patterns alters leaf vein architecture in Arabidopsis thaliana. Planta, 226, 1207-1218.

Laguna, M.F., Bohn, S. and Jagla, E.A. (2008) The role of elastic stresses on leaf venation morphogenesis. PLoS Comput. Biol. 4, e1000055.

Meinhardt, H. (1976) Morphogenesis of lines and nets. Differentiation, 6, 117123

Merks, R.M.H., Brodsky, S.V., Goligorksy, M.S., Newman, S.A. and Glazier, J.A. (2006) Cell elongation is key to in silico replication of in vitro vasculogenesis and subsequent remodeling. Dev. Biol. 289, 44-54.

Merks, R.M.H., Van de Peer, Y., Inzé, D. and Beemster, G.T.S. (2007) Canalization without flux sensors: a traveling-wave hypothesis. Trends Plant Sci. 12, 384-390.

Mezentzev, A., Merks, R.M.H., O'Riordan, E., Chen, J., Mendelev, N., Goligorsky, M.S. and Brodsky, S.V. (2005) Endothelial microparticles affect angiogenesis in vitro: role of oxidative stress. Am. J. Physiol. 289, H1106-H1114.

Price, C.A., Symonova, O., Mileyko, Y., Hilley, T. and Weitz, J.S. (2011) Leaf extraction and analysis framework graphical user interface: segmenting and analyzing the structure of leaf veins and areoles. Plant Physiol. 155 236-245.

Qi, R. and John, P.C.L. (2007) Expression of genomic AtCYCD2;1 in Arabidopsis induces cell division at smaller cell sizes: implications for the control of plant growth. Plant Physiol. 144, 1587-1597.

Robles, P., Fleury, D., Candela, H. et al. (2010) The RON1/FRY $1 / S A L 1$ gene is required for leaf morphogenesis and venation patterning in Arabidopsis. Plant Physiol. 152, 1357-1372.

Rolland-Lagan, A.-G. and Prusinkiewicz, P. (2005) Reviewing models of auxin canalization in the context of leaf vein pattern formation in Arabidopsis. Plant J. 44, 854-865.

Rolland-Lagan, A.-G., Amin, M. and Pakulska, M. (2009) Quantifying lea venation patterns: two-dimensional maps. Plant J. 57, 195-205.

Runions, A., Fuhrer, M., Lane, B., Federl, P., Rolland-Lagan, A.-G. and Prusinkiewicz, P. (2005) Modeling and visualization of leaf venation patterns. ACM Trans. Graph. 24, 702-711.

Scarpella, E. and Meijer, A.H. (2004) Pattern formation in the vascular system of monocot and dicot plant species. New Phytol. 164, 209-242.

Scarpella, E., Francis, P. and Berleth, T. (2004) Stage-specific markers define early steps of procambium development in Arabidopsis leaves and correlate termination of vein formation with mesophyll differentiation. Development, 131, 3445-3455.

Scarpella, E., Marcos, D., Friml, J. and Berleth, T. (2006) Control of leaf vascular patterning by polar auxin transport. Genes Dev. 20, 1015-1027.

Sieburth, L.E. and Deyholos, M.K. (2006) Vascular development: the long and winding road. Curr. Opin. Plant Biol. 9, 48-54.

Steynen, Q.J. and Schultz, E.A. (2003) The FORKED genes are essential fo distal vein meeting in Arabidopsis. Development, 130, 4695-4708.

Wenzel, C.L., Schuetz, M., Yu, Q. and Mattsson, J. (2007) Dynamics of MONOPTEROS and PIN-FORMED1 expression during leaf vein pattern formation in Arabidopsis thaliana. Plant J. 49, 387-398. 